Jens Ilg*

\title{
Mehr Spielräume: Methoden der partizipativen Lernraumgestaltung
}

DOI 10.1515/bfp-2016-0064

Zusammenfassung: Das an der UB Rostock realisierte und 2016 mit „Zukunftsgestalter in Bibliotheken 2016“ prämierte Projekt „Lernraum Bibliothek 2015“ war ein auf zwei Jahre angelegtes Methodenprojekt, in dem mit Methoden der partizipativen Nutzerforschung experimentiert wurde, inwieweit sie sich auch für die Lernraumgestaltung und -forschung eignen. Der Artikel beschreibt sieben dieser Methoden, gegliedert nach Zweck, Vorgehensweise, Vorteile, Risiken und Praxistipps.

Schlüsselwörter: Lernraum; Methoden; partizipatives Gestalten

\section{Creative Leeways: Procedures to Design Learning Spaces}

Abstract: In 2016 the Rostock University Library finished the project Library as a Learning Space 2015, a project which has been experimenting with procedures of participatory design to design learning spaces. The article describes seven procedures, which were successful in Library as a Learning Space 2015.

Keywords: Learning space; procedures; participatory design

Inhalt

1 „Lernraum Bibliothek 2015“ der UB Rostock . . 347

1.1 Lernraum? . . . . . . . . . . . . . . . . . . . . . . 348

1.2 Wozu Lernraumforschung? . . . . . . . . . . 348

2 Methoden der partizipativen Lernraumgestaltung 349

2.1 Walking in your customers shoes . . . . . . . 349

2.2 Innenarchitekt auf Zeit . . . . . . . . . . 350

2.3 Sag's farbig! . . . . . . . . . . . . 352

2.4 Good-Practise-Reise für Bibliotheksnutzer . . . 353

2.5 Lerngedichtwettbewerb . . . . . . . . . . . 355

2.6 Design-Workshops ................ . 356

2.7 Möbelcasting. . . . . . . . . . . . . 358

3 Fazit .................... 360

*Kontaktperson: Jens Ilg, jens.ilg@uni-rostock.de

\section{1 „Lernraum Bibliothek 2015“ der UB Rostock}

Das an der UB Rostock realisierte und geförderte Drittmittelprojekt „Lernraum Bibliothek 2015“ wurde vergangenes Jahr abgeschlossen (Laufzeit 2013 bis 2015). Es diente zum einen der Planungsvorbereitung für einen anstehenden Bibliotheksneubau, zum anderen war es - und das in erster Linie - ein Methodenprojekt, mit dem mit bekannten und neu entwickelten Methoden aus der partizipativen Nutzerforschung experimentiert wurde, inwieweit sie sich auch für die Lernraumgestaltung und -forschung vor Ort eignen: Der Weg war das Ziel.

In den Projektplan eingebaut waren zwei Besonderheiten:

- Partizipatives Gestalten ${ }^{1}$ : Konsequent wurde auf den Ansatz des Partizipativen Gestaltens gesetzt, d. h., diejenigen, für die die Lernräume künftig oder gegenwärtig gestaltet werden sollen, wurden aktiv einbezogen und haben so den Lernraum empirisch (z. B. in Form von Befragungen), konzeptionell oder gestalterisch maßgeblich mitverändert. Das waren vorwiegend studentische Bibliotheksnutzer, die die UB Rostock z.B. in Design-Workshops, Zonierungsprojekte, Möbelcastings oder als Innenarchitekten auf Zeit eingebunden hat (siehe Abschnitt 2).

- Bibliotheksnutzer als Projektmitarbeiter: Studentische Bibliotheksnutzer wurden zudem als Mitarbeiter für dieses Projekt engagiert; im Umfang von insgesamt ca. einer VZÄ waren stets fünf bis sechs Studierende im Projektteam. Neben organisatorischen und Projektumsetzungsaufgaben war deren Aufgabe, a) Brücken zu schlagen, d.h., als peer to peer waren sie in der Funktion eines Moderators in Workshops mit Studierenden oder sie hatten die Funktion eines Networkers, der sich mit anderen studentischen Bibliotheksnutzern vernetzt, um sie als Probanden oder als Interviewpartner $\mathrm{zu}$ gewinnen. Zudem hatten sie b) die

1 Zu einer begrifflichen Einordnung siehe hier Ilg und Zepf (2016). 
Aufgabe, ein erstes Testfeedback zu geben, wenn z.B. eine Idee für eine konkrete (Lernraum-)Service-Erweiterung entstand, wurden sie quasi stellvertretend für andere studentische Bibliotheksnutzer um ihre (nicht repräsentative) Einschätzung gebeten. Schließlich und nicht zuletzt waren sie auch c) Ideengeber, die neue, fruchtbare und gelegentlich unorthodoxe Lösungsansätze ins Spiel brachten.

- Diese projektorganisatorischen Besonderheiten waren mitverantwortlich dafür, dass weitere interessante Projektideen entstanden und das gesamte Projektteam beflügelten - mit dem Ergebnis, dass aus den ursprünglich neun geplanten Teilprojekten 33 ergiebige wurden. Die Spannbreite der Teilprojekte reicht u.a. von Befragungen von studentischen Eltern über Teststellungen von Wasserspendern und Arbeitsmobiliar bis zu einem Zonierungskonzept und der Einführung eines Feedbackmanagements. Der Preis „Zukunftsgestalter in Bibliotheken 2016“ für dieses Projekt geht damit auch wesentlich auf die studentischen Projektmitarbeiter zurück und er ist zugleich einer für die Bibliotheksnutzer der UB Rostock, die sich aktiv beteiligten.

\subsection{Lernraum?}

Bevor eine Auswahl von Methoden der Lernraumgestaltung beschrieben wird, hilft eine Definition, für was hier Lernraum steht. Der Lernraum Hochschulbibliothek wird nicht beschreibend (deskriptiv), sondern als Aufforderung (normativ) definiert: Die Hochschulbibliothek als Lernraum zu begreifen, heißt, ihre Services und räumlichen Bedingungen so $\mathrm{zu}$ gestalten, dass sie bestmöglich die Lern- und Arbeitsleistungen ihrer Nutzer fördern können.

Lerntheoretisch gehören Bibliotheksservices und -arbeitsplätze zur s.g. Lernumgebung. Die Zunahme der Bedeutung dieser Lernumgebung steht wiederum vor dem Hintergrund mindestens zweier Entwicklungen: Das ist zum einen die Bologna-Reform, die u.a. das (studentische) Selbststudium in den Mittelpunkt gerückt hat: Nutzen heute Studierende für ihre Selbststudienphasen (Vor-, Nachbereitung Seminare, Erstellen von Hausarbeiten, Referate, Lernen für Klausuren u.a.m.) ihren Universitätscampus, dann nutzen sie dafür durchschnittlich in der Hälfte dieser Zeit ihre Universitätsbibliothek (das gilt für fast alle Fächer), so eine Untersuchung der HIS GmbH. ${ }^{2}$ Zum anderen

2 Die Untersuchung bezieht sich nur auf die Vorlesungszeit, so dass mutmaßlich der Wert für das gesamte akademische Jahr höher liegt. Vgl. Vogel und Woisch $(2013,27)$. gehört zum Hintergrund eine Art Spatial Turn, d.h. ein im Grunde schon lange sich andeutender Trend der Zuwendung zum physischen Ort (parallel zum virtuellen), der auch im Lebensalltag sichtbar wird, z. B. im stationären Einzelhandel, in dem mit Lounge-Ecken im Supermarkt oder mit stimmungsvollen Interieur in Bäckereien auf deutlich mehr Aufenthaltsqualität gesetzt wird.

Die Bedürfnisse und Erwartungen gegenwärtiger Bibliotheksnutzer an die Hochschulbibliothek haben sich verändert, erste Indizien dafür werden vielerorts bereits wahrgenommen, z.B. längere Verweildauer, hohe Nachfrage nach lernunterstützenden Beratungen (z. B. in Gestalt der Langen Nächte der aufgeschobenen Hausarbeiten), Zunahme von Gruppenarbeiten.

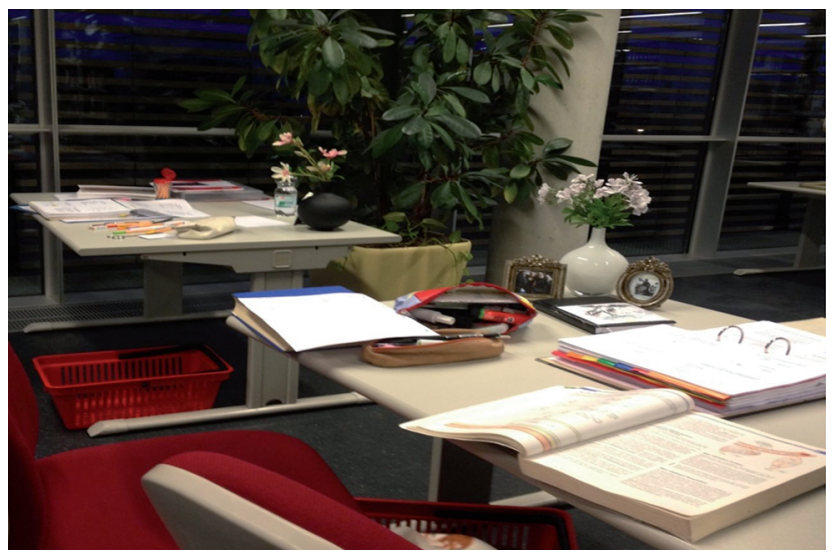

Abb. 1: Schnappschuss eines Nutzerarbeitsplatzes in der UB Rostock: Bibliotheksnutzer haben es sich behaglich gemacht mit selbstmitgebrachten Kunstblumen, Vasen und Fotos

\subsection{Wozu Lernraumforschung?}

Diese Entwicklungen sind Grund genug, Hochschulbibliotheken zu Lernräumen weiterzuentwickeln, und zwar begleitet von eigenen, lokalen Lernraumforschungen, wofür auch diese Gründe sprechen:

- Expertiselücke: Die Gestaltung des Lernraums Hochschulbibliothek erstreckt sich auch - aber keinesfalls nur - auf die Bibliothek als physischen Raum, was bspw. die Gestaltung von Nutzerarbeitsplätzen, Servicetheken, Schulungsräumen (Teaching Library) ebenso umfasst wie Lärmmanagement, Gebäudenavigations-, Beleuchtungs- oder Farbkonzepte. Da Pädagogische Architektur, Lerntheorien, Raumpsychologie o. ä. zumeist nicht wesentlicher Bestandteil bibliothekarischer Ausbildungen war, fehlt an nicht wenigen Hochschulbibliotheken Lernraumgestaltungsexpertise. Neben entsprechenden strukturell-organisatorischen Anpassungen trägt eigene Lernraumforschung 
wesentlich dazu bei, diese Expertiselücke zu verkleinern.

- Sensibilisierung: Die Lernraumorientierung von Bibliotheknutzern und damit deren veränderten Bedürfnisse sind ein vergleichsweises junges Phänomen: Geht man von ersten Untersuchungen und überregionalen Beobachtungen aus, scheint daraus eine gestiegene Nachfrage entstanden zu sein, z.B. für stationäre PC-Arbeitsplätze, den klassischen Lesesaal (neben Gruppenarbeitsplatzangeboten), Power-Nap-Angebote oder bewegtes Lernen (bewegungsförderliche Arbeitsplatzangebote). Eigene Lernraumforschung hilft, Mitarbeiter an Hochschulbibliotheken und deren Beschaffungsstellen dafür zu sensibilisieren, dass solche Bedürfnisse nicht mehr abwegig sind.

- Nutzer verstehen lernen: Ein wesentliches Ziel von Nutzer- und damit Lernraumforschung ist, so auf ein klares Bild von aktuellen Nutzerbedürfnissen zu stoßen. Das gilt unabhängig davon, ob die Ergebnisse gegenwärtig oder künftig vor Ort realisierbar sind.

\section{Methoden der partizipativen Lernraumgestaltung}

Lernräume an Hochschulbibliotheken lassen sich vielfältig partizipativ erkunden und gestalten. Es folgt ein Überblick über Methoden, die sich im o.g. Projekt bewährt haben und die geeignet scheinen, Lernraumforschungen an anderen Hochschulbibliotheken anzuregen und ggf. angepasst auf sie zu übertragen.

Die Übersicht gibt die Erfahrungen und das Verständnis der Methoden wieder, wie sie im o.g. Projekt definiert und eingesetzt wurden; ein Anspruch auf allgemeingültige Darstellung erhebt sie nicht.

\subsection{Walking in your customers shoes}

\subsubsection{Zweck}

Diese Methode wird eingesetzt, um die eigene Bibliothek als physischen Ort aus Perspektive ihrer Nutzer zu erkunden. Die methodische Fragestellung lautet allgemein: Was nehmen unsere Nutzer wahr, was wir möglicherweise nicht oder nicht mehr wahrnehmen?

\subsubsection{Methodischer Kerngedanke}

Unsere je eigene Bibliothek mit ihren Services und als physischer Ort ist uns nicht selten nur scheinbar bekannt. Häufig tendieren wir unweigerlich zu einer Art Tunnelblick: Wir nehmen das Setting aus Services, Nutzerarbeitsplätzen, Servicetheken und Laufwegen gewohnheitsmäßig und damit möglicherweise nur unvollständig wahr. Und wir nehmen sie zudem möglicherweise nur aus Sicht eines Professionals wahr, nicht aber mit den Augen der eigenen aktuellen Bibliotheksnutzer, die sich zudem - anders als wir - nur temporär dort aufhalten. Diese Methode kann unsere Sicht erweitern und ergänzen.

\subsubsection{Vorgehensweise}

Um einen unverstellten Blick von außen zu gewinnen, sollten als Walker weder Professionals anderer Bibliotheken noch eigene Stammbibliothekskunden eingesetzt werden. Letztere sind mit den Services und dem Gebäude schon zu vertraut oder sie haben sich - für sie unmerklich - mit den möglicherweise defizitären und auffälligen Gegebenheiten arrangiert. Gut geeignet sind mehrere fremde oder eigene Studienanfänger. Für den internen Bereich (BackOffice) eignen sich neue Kollegen. Sobald die Walker feststehen, geht man im einfachsten Fall so vor: Nachdem die Bibliotheksleitung ihr Einverständnis gegeben hat, besuchen und nutzen sie die fragliche Bibliothek (geschlossen oder getrennt) an einem oder mehreren Tagen: Sie nutzen sie in der Rolle eines Bibliotheksnutzers unvoreingenommen, aufmerksam und lassen sie sich systematisch auffällig werden anhand von zuvor formulierten Leitszenarien. Ausgestattet mit Fotoapparat und Schreibmaterial lassen die Walker sich auf die dortigen Services und räumlichen Gegebenheiten ein und folgen einem zuvor eigenständig (!) formulierten typischen Nutzungsszenario, z. B. dem folgenden Szenario: „Nutze stationäre PC-Arbeitsplätze zur Suche nach Büchern an diesem Standort: Gibt es PC-Arbeitsplätze auch in ruhigen Arealen? Ist die Nutzung des Katalogs mit diesem Rechercheziel ohne Hilfe und Bratung möglich?“. Die Erfahrungs- und Beobachtungsergebnisse dieser Testnutzungen werden schriftlich dokumentiert, und zwar am besten mit Bezug auf den Raumplan der Bibliothek. Die anschließende Auswertung durch die Walker basiert auf einer getrennten Dokumentation, d.h., das Beobachtete und Erfahrene (inkl. Foto) wird getrennt dargestellt von deren Deutung. Im Idealfall liefern die Walker zudem auch Lösungsanregungen, sofern aus deren Sicht Handlungsbedarf attestiert wurde. 
Diese Methode ist gut geeignet, um komplette Bibliotheksnutzungsvorgänge und deren Abstimmungsgrad aufeinander zu prüfen, z. B. vom Suchen des Haupteingangs über das Ablegen von Garderobe und Suche nach Gebäudeinformationen (Leitsystem) bis zum Aufsuchen von Servicepersonal, Nutzung eines Katalogs und Auschecken von Büchern.

\subsubsection{Vorteile}

Für diese Methode spricht v.a. die Unvoreingenommenheit, mit der die Walker beobachten und testnutzen. Deren Ergebnisse ergänzen das je eigene Bild der Professionals vor Ort und tragen nachhaltig zur Relativierung deren Eigenwahrnehmung von Standort und Services bei.

Nicht weniger vorteilhaft ist der so leicht mögliche ganzheitliche Blick der Walker: Die Testnutzungen können komplette (typische) Nutzungsszenarien durchspielen, d.h., es werden nicht einzelne Services probiert, sondern alle Schritte, die Bibliotheksnutzer gehen müssen, um z. B. ein bestimmtes Buch auszuleihen: vom Betreten der Bibliothek über die Orientierung im Gebäude und der Literaturrecherche bis zum Regalbrowsing, Buchauschecken und Laufweg zu einem ÖPNV-Haltepunkt.

\subsubsection{Risiken}

Mystery Shopping: Da die Walker eine Testnutzung durchspielen (Nutzungsleitszenarien), besteht die Gefahr, die Grenze zum Mystery Shopping zu übertreten, also zu einer verdeckten Testnutzung, mit der die Kompetenzen und Arbeitsqualitäten des Bibliothekspersonals geprüft wird. Das wird vermieden durch eine eindeutige Auftragsbeschreibung an die Walker (die das explizit ausschließt) sowie eine transparente Kommunikation gegenüber den Kollegen vor Ort.

Ergebnisrelativierung: $\mathrm{Zu}$ den Ergebnissen können räumliche oder bauliche Defizite gehören, die vor Ort entweder bereits bekannt sind und/oder die sich aus deren Sicht nicht aufheben lassen, z.B. fehlende barrierefreie Brandschutztüren, fehlende Verstromung von Nutzerarbeitsplätzen. Diese Erwartung sollte nicht der Grund sein, den Einsatz dieser Methode nicht zu erwägen. Empfehlenswert ist eine grundsätzlich offene Haltung gegenüber den Ertrag dieser Methodenanwendung vor Ort und die Bereitschaft, die technischen oder räumlichen Gegebenheiten und die Bibliotheksservices nicht als alternativlos $\mathrm{zu}$ betrachten, sondern sie auf den Prüfstand und infrage zu stellen. Und auch wenn sich beobachtete Defizi- te kurz- und mittelfristig nicht beheben lassen, sie lassen sich i.d.R. gegenüber Bibliotheksnutzern verständniswerbend kommunizieren, wofür sie aber erst bekannt sein müssen. Zum Ertrag dieser Methode gehören zudem häufig andere wichtige Beobachtungen, die ohne diese Walks nicht bekannt werden würden.

\subsection{Innenarchitekt auf Zeit}

\subsubsection{Zweck}

Innenarchitekt auf Zeit meint, dass Bibliotheksnutzer einen Raum (oder mehrere) eigenverantwortlich und so komplett wie möglich zu einem aus deren Sicht lern- und arbeitsförderlichen Raum neu oder umgestalten: Sie entwickeln eine Lernraumgestaltungskonzeption für einen konkreten Raum, die die Hochschulbibliothek anschließend möglichst ohne Abstriche umsetzt. Die, die den Raum zum Lernen und Arbeiten nutzen, sind auch die, die ihn für diese Funktion gestalten und somit temporär in die Rolle eines Innenarchitekten schlüpfen.

\subsubsection{Methodischer Kerngedanke}

Innenarchitekt auf Zeit ist eine Erweiterung der Methode Design-Workshops (siehe unten): Sie umfasst drei Etappen der Lernraumnutzerforschung, d.h., neben der a) Erhebung von Bedürfnissen an den Lernraum Bibliothek umfasst sie auch b) deren Übersetzung in ein entsprechendes realisierbares (Lernraum-)Gestaltungskonzept, einschließlich c) dessen Realisierung und Evaluation. Der Charme dieser Methode liegt darin, dass die, deren Lernraumbedürfnisse hiermit implizit erhoben werden, auch die sind, die sie in die ihres Erachtens passende Lernraumlösungen ummünzen. Und sie bietet aus Sicht von Bibliotheksnutzern zwei starke Anreize, sich (freiwillig) zu beteiligen: die Umsetzungsgarantie und einen Lernraum weitgehend selbst gestalten zu können.

\subsubsection{Vorgehensweise}

Am Beginn steht die Festlegung des Gestaltungsrahmens durch die Hochschulbibliothek, d. h.

- Festlegung eines Raumes (oder Räume, Flächen), der Lernraumpotenzial hat und dafür bisher nicht aktiviert wurde.

- Festlegung der Gestaltungsaufgabe, d.h. Festlegung, was dort neu gestaltet werden kann (z. B. Neumöblie- 
rung, neue Deckenbeleuchtung, Begrünung mit Zimmerpflanzen), und was dort nicht neu gestaltet werden kann (z. B. keine Veränderung von Bediengangund Fluchtwegbreiten, Neuverkabelung von LAN- und Stromanschlüssen). Beides sollte mit dem Gebäudemanagement und Sicherheitsbeauftragten zuvor abgestimmt werden.

- Festlegung des Beschaffungsetats (z. B. für Bepflanzung, Beleuchtung, Mobiliar).

- projektorganisatorische Festlegungen (z.B. bibliotheksseitige Projektgruppe, Planungs-, Umsetzungszeitraum, Arbeitspakete).

Anschließend gilt es, dafür interessierte Bibliotheksnutzer zu gewinnen; neben den methodischen Anreizen (Umsetzungsgarantie, Lernraum selbst gestalten) können materielle Anreize wie Gutscheine für Einrichtungshäuser oder für Bücher helfen. Die akquirierten Bibliotheksnutzer werden anschließend eingewiesen in die

- Gestaltungsaufgabe,

- allgemeinen Gestaltungsmöglichkeiten von Lernräumen (z.B. in Rahmen eines Kurzworkshops, in dem aktuelle Lernräume anderer Einrichtungen oder mobiliare Lösungen für Lern- und Arbeitsumgebungen präsentiert werden),

- eigenen Nutzerforschungs- bzw. Lernraumergebnisse (sofern vorhanden).

Daran schließt sich die eigentliche kreative Phase an: Jene Innenarchitekten bekommen nun Zeit (z. B. 2 bis 4 Wochen) und, sofern möglich, einen Arbeitsraum, um selbstständig eine Gestaltungs- sowie eine Evaluationskonzeption $\mathrm{zu}$ entwickeln. Die Evaluation beginnt nach der Realisierung und animiert andere Bibliotheksnutzer $\mathrm{zu}$ Rückmeldungen zum neu realisierten Lernraum.

Die bibliotheksseitige Projektgruppe steht währenddessen auf Abruf für Rückfragen oder Beratungen zur Seite (z.B. bei Fragen zu Farbharmonien, betrieblichen Abläufen); weitere gestalterische Gebote jedoch gibt sie nicht.

Das Gestaltungskonzept wird schließlich von der bibliotheksseitigen Projektgruppe geprüft, inwieweit sie den o.g. Festlegungen entsprechen. Im Anschluss wird das Konzept, inkl. Evaluationskonzept in Abstimmung mit dem a) Gebäudemanagement, b) Sicherheitsbeauftragen und c) Möbelausstattern operationalisiert und realisiert. Nach der Realisierung wird der neu gestaltete Lernraum evaluiert, d.h., Bibliotheksnutzer bekommen Gelegenheit zu einem Feedback, auch um erwägen zu können, diese Gestaltungskonzeption auf andere vergleichbare Räume oder Standorte zu übertragen.
Diese Methode ist auch gut geeignet zur Aktivierung potenzieller Lernräume, die bisher nicht als solche genutzt wurden.

\subsubsection{Vorteile}

Besonders für diese Methode spricht, dass die von Bibliotheksnutzern selbst entwickelten Lernräume in der Regel überdurchschnittlich hoch akzeptiert werden, nicht zuletzt deshalb, weil sie ihre Bedürfnisse zugleich in Gestaltungslösungen übersetzen konnten. Nicht weniger attraktiv macht sie der methodisch verbundene Zwang zur Realisierbarkeit, d.h., am Ende stehen nicht nur Lösungen auf dem Papier, sondern auch im Raum. Und ein nicht unerwünschter Nebeneffekt ist zudem, dass die häufig beobachtete ,wilde Gestaltungsenergie، von Bibliotheksnutzern (z.B. unerlaubtes Verräumen von Mobiliar) in positive, bibliotheksgelenkte Gestaltungsenergie kanalisiert wird.

\subsubsection{Projektrisiken}

Der Ertrag mit dieser Methode gerät besonders dann in Gefahr, wenn die bibliotheksseitige Offenheit für unorthodoxe Lösungen und der Gestaltungsspielraum zu klein gehalten wird: Das verringert die Gestaltungskreativität und den Anreiz für Bibliotheksnutzer, daran mitwirken zu wollen. Weniger gefährdend ist der Umstand der fehlenden Repräsentativität der Gestaltungslösung, die von wenigen Bibliotheksnutzern entwickelt wird und schließlich von vielen anderen genutzt werden soll: Diese fehlte vermutlich bereits, denn in der Regel basieren die innenarchitektonischen Konzepte in vielen Hochschulbibliotheken nicht auf repräsentativen Abstimmungsverfahren o.ä., sondern vielmehr auf Entwürfen eines Architektur- bzw. Planungsbüros oder auf den mutmaßlich kleinsten gemeinsamen ästhetischen und funktionalen Nenner. Die mit dieser Methode möglichen Gestaltungslösungen können einen attraktiven Kontrapunkt setzen. Die an der UB Rostock aktiven Innenarchitekten auf Zeit haben weder egoistische noch unrealistische Gestaltungslösungen entwickelt.

\subsubsection{Praxistipps}

- Nutzerakquise: Die Innenarchitekten auf Zeit sollten ein Mix von Vertretern der erwarteten Bibliotheksnutzer sein; zu ihnen sollten auch Menschen mit körperlichen oder psycho-somatischen Beeinträchtigungen gehören. 
- Beschaffung: Weil nicht standardisierte Möbellösungen entstehen können, empfiehlt sich, dieses Projekt zuvor mit der Beschaffungsstelle o. ä. und Gebäudemanagement abzustimmen.

- Lokale Nutzerforschungen: Im optimalen Fall liegen eigene (oder übertragbare) Beobachtungen und Ergebnisse von Nutzerforschungen vor, auf die die Konzeption der Innenarchitekten auf Zeit aufbauen kann.

- Gestaltungsspielräume: Das Wissen um die Spannbreite von Möglichkeiten für mobiliare Raumlösungen kann z. B. durch Besuch von Ausstattungsmessen wie die Orgatec erweitert und aktuell gehalten werden.

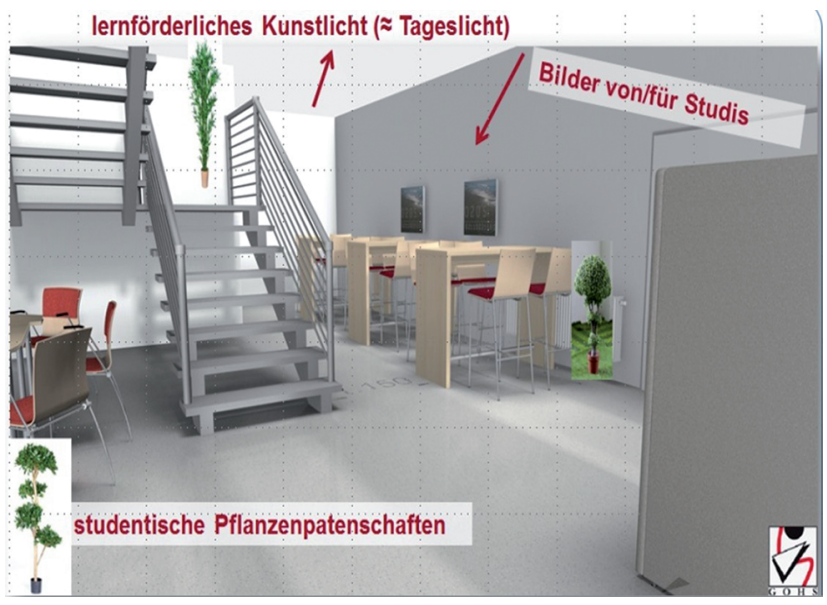

Abb. 2: Innenarchitekten auf Zeit an der UB Rostock: Gestaltungskonzept für einen bisher nichtaktivierten Raum

\subsection{Sag's farbig!}

\subsubsection{Zweck}

Sag's farbig! ist eine (nicht repräsentative) kreative Rückmeldungsmethode, die Bibliotheksnutzern ermöglicht, niedrigschwellig und rasch Feedback zu Services, Raumlösungen, Arbeitsplatzausstattung u.a. formulieren zu können.

\subsubsection{Methodischer Kerngedanke}

Aufgrund der einladenden Niedrigschwelligkeit wird der Kreis der Rückmeldenden erweitert um Bibliotheksnutzer, für die herkömmliche Rückmeldungsangebote (z. B. ,Kummerkasten', Webformular, mündlich an Servicetheken) als zu umständlich gelten.

\subsubsection{Vorgehensweise}

Dieses für Bibliotheken vergleichsweise nicht besonders aufwändige Evaluations- und Nutzerforschungsinstrument orientiert sich am Prinzip Abstimmung (Voting). Benötigt wird eine Einwurfbox (z.B. Karton mit Einwurfschlitz), drei Notizzettelstapel in den Farben grün, gelb, rot sowie Stift und ein Aushang, der kurz beschreibt, wie und für was zum Feedback eingeladen wird. Der rote Zettelstapel steht z. B. für „Finde ich nicht so gut!“, der gelbe für „teils/teils“, der grüne für „Finde ich sehr gut!“. Über oder neben diesen Stapeln wird zu verbalen Rückmeldungen mit einladenden Vervollständigungsfragen ermuntert, z.B. „Wie findest du es, dass ...? Das finde ich ...“. Das Setting aus Box, Stapel, Stift und Aushang wird anschließend zusammen mit dem, was evaluiert werden soll, an einem geeigneten Raumpunkt postiert. Die Bibliotheksnutzer haben im Vorbeigehen Gelegenheit, zu votieren und wenn sie mögen - auf jene Zettel zusätzlich Kommentare zu notieren, wovon häufig Gebrauch gemacht wird (s. auch Abb. 3).

Diese Methode ist auch gut geeignet für rasch benötigtes Feedback zu Möbeltests, zu neuen Services und als Alternative zu einem Ausstellungs-Gästebuch.

\subsubsection{Vorteile}

Der damit mögliche hohe Rücklauf macht dieses Instrument attraktiv: In kurzer Zeit sind viele Voten und verbale Rückmeldungen möglich, die sich zudem bibliotheksseitig vergleichsweise leicht erfassen und auswerten lassen. Es provoziert wesentlich stärker zu Rückmeldungen als es mit herkömmlichen Feedbackoptionen in der Regel möglich ist.

\subsubsection{Risiken}

Die Niedrigschwelligkeit hat auch eine Kehrseite: Sie kann Bibliotheksnutzer zu Nonsenskommentaren und anderen nicht intendierten Statements verleiten (z.B. politische Statements, Unmutsbekundungen). Die geringe Spannbreite von Votingoptionen (Farben rot, gelb, grün) macht das Instrument zudem ungeeignet für differenziertes Feedback. 


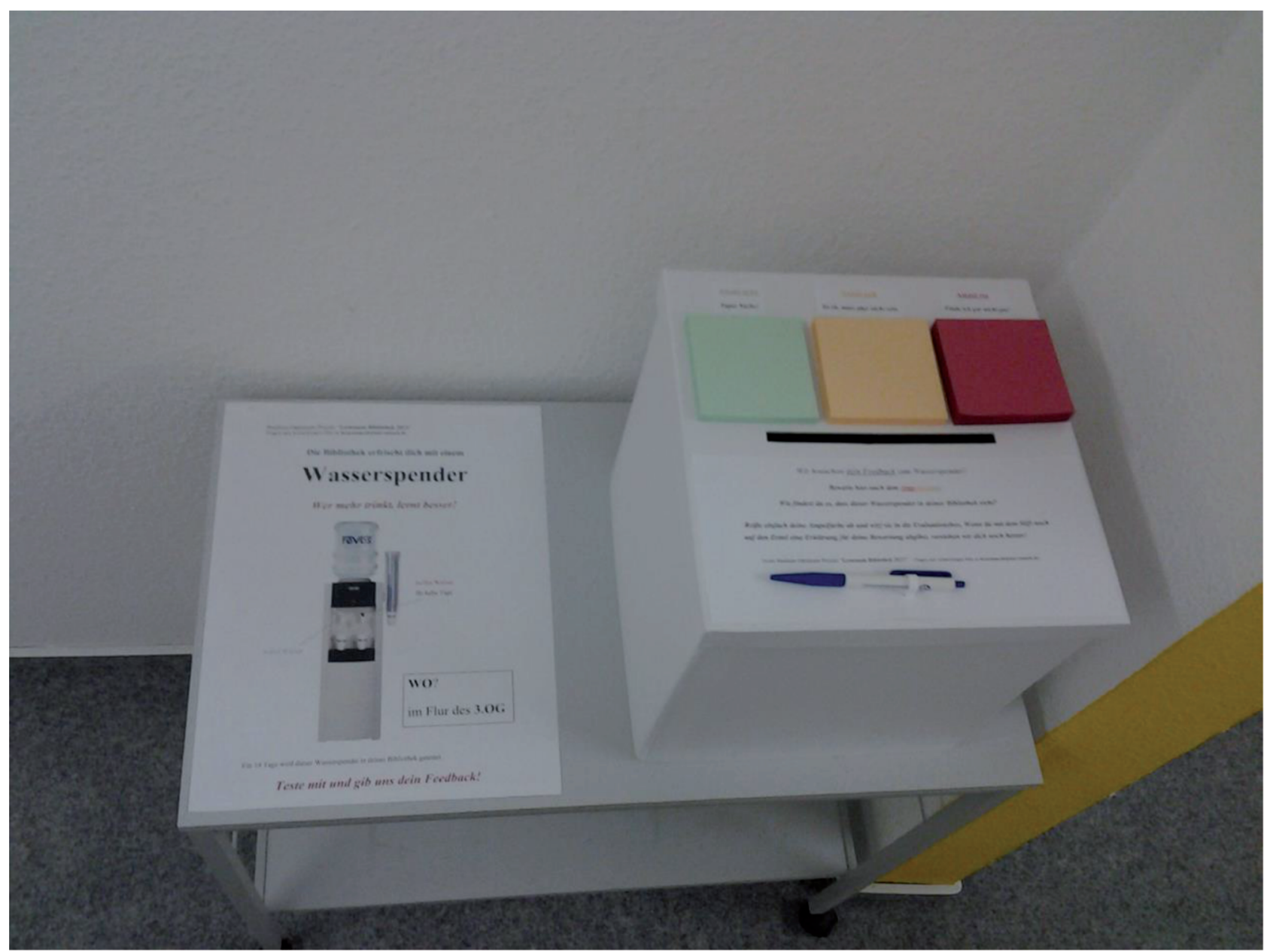

Abb. 3: Mit „Sag’s farbig!“ geben Bibliotheksnutzer Feedback zu einem testweise aufgestellten Wasserspender an der UB Rostock

\subsubsection{Praxistipp}

Empfohlen wird, mit nicht mehr als drei Abstimmungsfarben zu arbeiten; mehr als drei macht das Instrument zu fehleranfällig und nutzungsunattraktiver.

\subsection{Good-Practise-Reise für Bibliotheksnutzer}

\subsubsection{Zweck}

Bibliotheksnutzer der eigenen Hochschulbibliothek werden auf selbstständige Erkundungsreise geschickt, andere beispielgebende oder anregende Hochschulbibliotheken zu nutzen und zu erkunden, um a) sich auf diese Weise die je eigenen Bedürfnisse und Erwartungen an den Lernraum (Bibliothek) bewusst zu machen, b) die Vielfalt von Lernraum- bzw. Bibliothekskonzepten kennenzulernen und c) Service- oder Gestaltungsideen für die eigene Hochschul- bibliothek zu entwickeln. Diese Methode ist auch gut geeignet in Kombination mit der oben beschriebenen Methode Innenarchitekt auf Zeit.

\subsubsection{Methodischer Kerngedanke}

Fragt man Bibliotheksnutzer, was sie von ihrer Hochschulbibliothek erwarten, basiert das häufig allein darauf, was sie bisher kennen, was in der Regel die eigenen Hochschul- und kommunalen Bibliotheken sind. Der Anteil der Bibliotheksnutzer, die bereits ein Auslandssemester absolviert oder zuvor an anderen Hochschulen studiert haben, ist gering. Hier kommen Good-PractiseReisen ins Spiel: Sie sind eine Art Kreativitätstechnik, mit der andere alternative Lernraumkonzepte, -ideen und -probleme in den Wahrnehmungshorizont geraten, mit denen wiederum die je eigene Hochschulbibliothek unweigerlich verglichen wird, was schließlich hilft, sich die je eigenen Lernraumbedürfnisse bewusster zu machen. 
Im Idealfall bringen die reisenden Bibliotheksnutzer eine Art Liste von Kriterien für einen guten bzw. schlechten Lernraum mit.

\subsubsection{Vorgehensweise}

Nachdem die bibliotheksinterne Projektgruppe sich auf die Finanzierung des Reisebudgets, die Anzahl der reisenden Bibliotheksnutzer, den Zeitraum und schließlich eine Auswahl von Hochschulbibliotheken, die bereist werden sollen, geeinigt hat, werden freiwillige Bibliotheksnutzer gesucht, die auf Reisen gehen möchten. Für sie wird die Reise organisiert und die $\mathrm{zu}$ besuchenden Bibliotheken über dieses Vorhaben informiert. Die Reisenden werden über ihren Reiseauftrag instruiert, der nicht aus Sicht bibliothekarischer Professionals und möglichst weit gefasst wird und (dennoch) einen ausreichend eindeutigen Beobachtungsauftrag beinhaltet. Die Reisenden der UB Rostock erhielten z.B. den Auftrag, die besuchten Bibliotheken zu den Zwecken nutzen, zu denen sie auch die UB Rostock nutzen, und dabei schriftlich alles das festzuhalten, was ihnen auffällt. Dabei galt es, sich stets die Frage zu stellen, ob sie dort gern/ungern lernen bzw. arbeiten würden und warum. Um die Unvoreingenommenheit nicht zu mindern, sollte eine Führung durch die besuchten Bibliotheken erst nach dem eigenen Vor-Ort-Studium folgen. Nach der Reise wird jenen Bibliotheksnutzern Zeit gegeben, die Eindrücke zu sortieren und sie abschließend der internen Projektgruppe zu präsentieren.

\subsubsection{Vorteile}

Dieses Verfahren ist im Vergleich zu den anderen hier beschriebenen nicht wesentlich aufwändiger und teurer, es bietet aber einen potenziell enormen Ertrag, der von einer Kriterienliste (wann ist was ein guter Lernraum) über Einblicke in andere Bibliothekskonzepte bis zu Gestaltungsideen und nicht zuletzt aktivierten Bibliotheksnutzern reichen kann. Die Erfahrungen an der UB Rostock haben gezeigt, dass diese Reisenden fortan hohes Mitwirkungsinteresse an der Weiterentwicklung ihres Lernraums haben. Auch für die besuchten Bibliotheken ist dieses Verfahren attraktiv, bietet es doch einen unvoreingenommen systematisch-analytischen Blick aus Nutzersicht auf ihre Bibliothek (vergleichbar mit der Methode „Walking ...“, siehe oben).

\subsubsection{Risiken}

Ein Risiko liegt in der Nutzerakquise: Nicht jeder Bibliotheksnutzer ist als Bibliotheksreisender geeignet. Erforderlich dafür ist eine Bereitschaft, selbstständig zu explorieren, also eigeninitiativ mit Neugierde die Zielbibliothek zu nutzen und sich anregen zu lassen. Ebenfalls kontraproduktiv kann die Formulierung des Beobachtungsauftrags werden: Wird er zu einem kleinteiligen Fragekatalog, ist er kaum von einer Besuchscheckliste zu unterscheiden. Das behindert einen unvoreingenommenen Blick und könnte genauso gut auch von beliebigen Personen vor Ort abgearbeitet werden.

\subsubsection{Praxistipps}

Nutzerakquise: Empfohlen wird eine Kleingruppe von Nutzern, die bereits Bibliotheken regelmäßig genutzt haben. Sind es studentische Bibliotheksnutzer, sollte der Zeitraum abhängig von der Vorlesungs- und Prüfungszeit gewählt werden. Wenige Reisetage sollten genügen.

- Auswahl Zielbibliotheken: Unter den zu besuchenden Bibliotheken sollten auch solche gehören, die sich in Art und Größe von der eigenen unterscheiden.

- Zusammenarbeit mit Studierenden in Austauschprogrammen: Ein Versuch ist es wert, Studierende der eigenen Hochschule, die temporär an ausländische Hochschulen wechseln, für eine solche Beobachtungsstudie zu gewinnen.

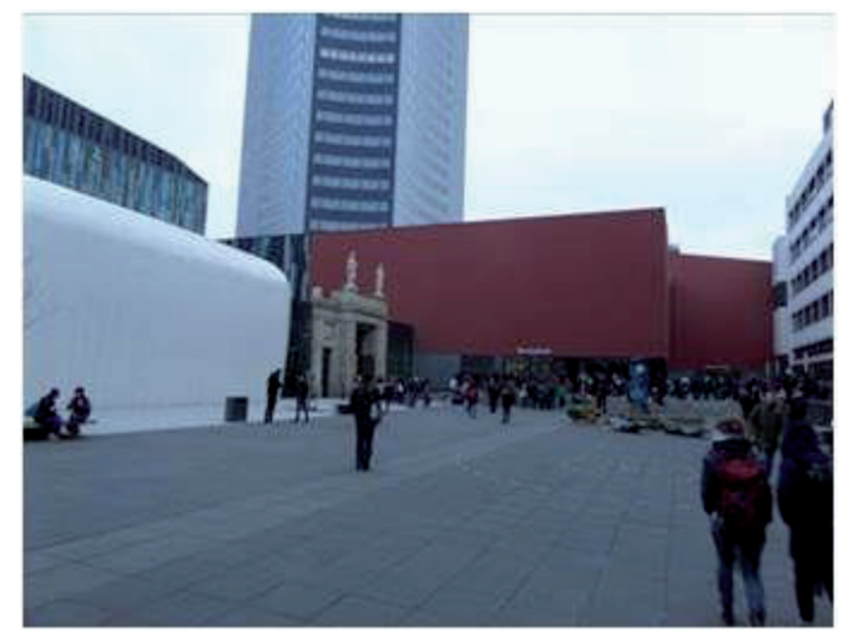

Abb. 4: Studentische Bibliotheksnutzer der UB Rostock besuchen einen Standort der UB Leipzig 


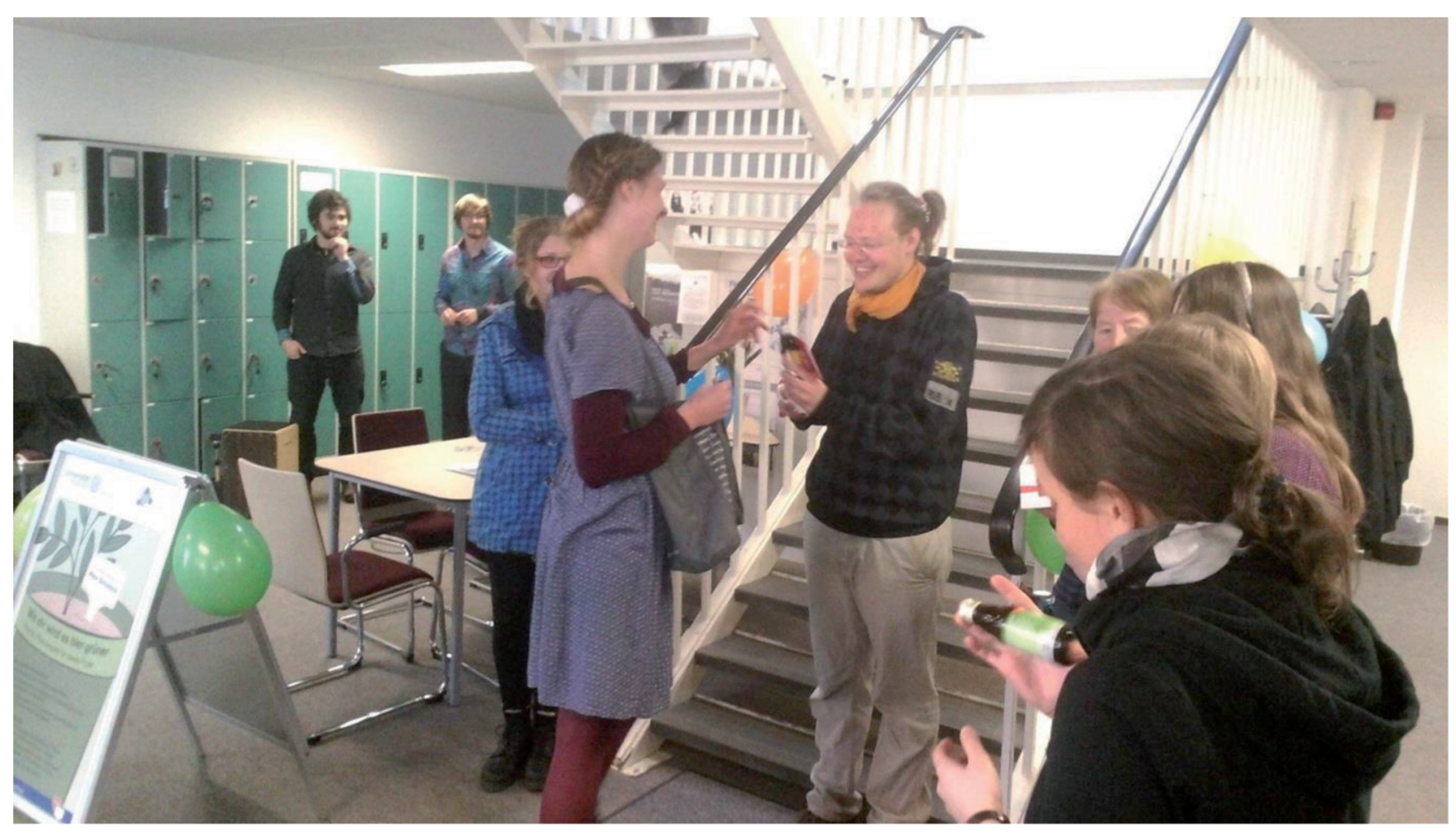

Abb. 5: Übergabe der Preise an die Gewinner im Lerngedichtwettbewerb „Dichte, was du lernst!“ der UB Rostock

\subsection{Lerngedichtwettbewerb}

\subsubsection{Zweck}

Lerngedichte sind hier mehrzeilige Reime, die inhaltlich entweder einen zu lernenden Stoff umfassen (z. B. Merksätze, Definitionen) oder sich auf das Lernen allgemein beziehen. Verbunden mit einem Wettbewerb (für Bibliotheksnutzer) dienen sie im Rahmen eines Lernraumprojekts als Begleitinstrument: Zum einen als Marketingmaßnahme, über das die Aufmerksamkeit auf ein bestehendes laufendes Projekt gelenkt wird, zum anderen als Sensibilisierungsmaßnahme, mit der die Sensibilität für lernförderliche Studienbedingungen erhöht werden kann.

\subsubsection{Methodischer Kerngedanke}

Ein Lerngedichtwettbewerb ist anders als die hier beschriebenen Methoden: Er dient nicht der Lernraumforschung, vielmehr hilft er, für ein Lernraumprojekt Öffentlichkeit herzustellen.

\subsubsection{Vorgehensweise}

Die Vorgehensweise unterscheidet sich grundsätzlich nicht von anderen Kreativitätswettbewerben wie Foto- oder Schreibwettbewerben: Am Beginn steht die Festlegung dessen, wann was ein Lerngedicht ist, die Festlegung der Teilnahmebedingungen, Teilnahmeanreize (z.B. Büchergutschein), Jury, Voting- und Einreicheverfahren, Wettbewerbszeitraum sowie der Art und Weise der Präsentation der Siegereinreichungen, inkl. Öffentlichkeitsarbeit.

\subsubsection{Vorteile}

Besonders wertvoll ist diese Methode aufgrund der Nebeneffekte: Zum einen können die hierüber entstehenden Reime zugleich als Fundus für gesuchte Slogans (Claims) dienen, ebenso für temporäre und von Bibliotheksnutzern kreierte Wandgestaltungen (z.B. in Form eines Plakatdrucks). In der UB Rostock z. B. zieren die Gewinnerlerngedichte mehrere bisher nur umständlich optisch aufwertbare Wände. Zum anderen werden Bibliotheksnutzer zu einer kreativen Lernpause zugunsten der Hochschulbibliothek animiert, die von den Ergebnissen dauerhaft zehren kann. 


\subsubsection{Risiken}

Die Erfolgsgefahr für dieses Instrument ist das Medium Gedicht: Für nicht wenige steht es für Hochkultur und nur wenigen mag das Reimen liegen. Hoher Rücklauf sollte nicht erwartet werden. Der Lerngedichtwettbewerb der UB Rostock erbrachte 12 relevante Gedichteinsendungen, die allerdings ausnahmslos alle von hoher Qualität waren, darunter auch Lerngedichte von Dozenten.

\subsubsection{Praxistipps}

- Nutzerakquise: Empfohlen wird, einen materiellen Teilnahmeanreiz zu schaffen, und zwar mit Lernraumbezug, z.B. Prämien wie E-Book-Reader, Kopfhörer, Einrichtungshausgutschein, Thermokanne, Radtasche.

- Jury: Auch um eine hohe Nutzerpartizipation zu ermöglichen, bietet sich an, über die Gewinner nicht durch eine Jury, sondern durch die gesamte Bibliotheksnutzerschaft entscheiden zu lassen, z. B. niedrigschwellig im Rahmen eines Blogs.

- Nutzungsrechte: Die Teilnahmebedingungen sollten weitreichende Nutzungsrechte für die Hochschulbibliothek beinhalten, z.B. das Recht zur Veröffentlichung auf eigenen Webseiten, Flyern etc.

\subsection{Design-Workshops}

\subsubsection{Zweck}

Zweck dieser Methode ist, die Erwartungen, Bedürfnisse und auch Ideen für den Lernraum Hochschulbibliothek zu ermitteln, indem Bibliotheksnutzer sie in Gestalt von Grundriss- bzw. Raumskizzen visualisieren. Ein DesignWorkshop ist zugleich eine Kreativitätstechnik, mit der deren Lernraumbedürfnisse in Ideen für Lernraumgestaltungen übersetzt werden können. Grundsätzlich lässt sich ein Design-Workshop auf alles Räumliche beziehen, z.B. auch auf Büros bzw. Mitarbeiterarbeitsplätze. An der UB Rostock wurde beides realisiert, für Studierende und Mitarbeiter.

\subsubsection{Methodischer Kerngedanke}

Design-Workshops im Kontext von Lernraumgestaltungsprojekten haben mehrere Pointen. Als Alternative zu Befragungen sind deren Ergebnisse quasi näher am Projektziel: Soll die Hochschulbibliothek nutzerorientiert partizipativ gestaltet werden, so entstünden damit (nutzerkommentierte) Visualisierungen bzw. Gestaltungskonzepte - anders als z.B. Befragungen, aus denen auf Basis von verbalen oder quantitativen Angaben anschließend Gestaltungskonzepte zu entwickeln sind. Zum anderen dienen DesignWorkshops auch als Kreativitätstechnik, die stärker als Befragungen oder als Walks (s. oben) helfen, sich eigene Lernraumbedürfnisse vor Augen zu führen und sie zugleich in Lösungen für Raumaufteilungen, Verkehrswege, Ausstattungen, Services, Farbkonzepte usf. umzumünzen. Das gelingt nicht zuletzt deshalb, weil methodisch die Bedingung, dass die Lösungen auch realisierbar sein müssen, entfällt.

\subsubsection{Vorgehensweise}

Auch diese Methode setzt auf die Mitarbeit von Bibliotheksnutzern: Nach einer knappen Einführung in Ziel und Methodik bekommen mehrere oder einzelne Bibliotheksnutzer in einem Kurzworkshop (insgesamt ca. 0,5 bis 1,5 h) die Gelegenheit, mittels Möbelschablonen, Schere, Stift u.ä. sowie anhand eines Grundrisses die Bibliothek so fiktiv umzugestalten, dass er ihren Bedürfnissen entspricht. Die Designer werden anschließend in einem Leitfrageninterview dazu animiert, ihre Skizzen zu kommentieren und ggf. das zu verbalisieren, was nicht visualisierbar war. Besonderer Wert auf den Verzicht der Realisierbarkeit gelegt wird, d.h., die Designer sollten sich nicht zugleich auch fragen, ob das grundsätzlich oder in ihrer Bibliothek eine Realisierbarkeitschance hätte.

Für die bibliotheksseitige Organisation eines solchen Vorhabens sind besonders wichtig:

- Nutzerakquise: Mit Design-Workshops lässt sich Repräsentativität nicht erreichen; dennoch hilfreich ist, von möglichst allen Zielgruppensegmenten Freiwillige zu gewinnen, z.B. auch aus verschiedenen Fachkulturen wie Geisteswissenschaftler, Sozialwissenschaftler. Da deren Teilnahme mit einem zeitlichen Aufwand verbunden ist, helfen zusätzliche Teilnahmeanreize. An der UB Rostock wurden z.B. Studierende face to face zwischen Lehrveranstaltungspausen angesprochen und dazu eingeladen, ihre Pause mit Kaffee, Kuchen und Radiomusik in den Design-Workshops zu verlegen.

- Moderation: Die Workshopleitung sollte sich der Moderationstechnik verpflichten, d.h., neben der Methoden- und Zielerläuterung gilt es, eine Beeinflussung bzw. Orientierung auf bestimmte Raumkonzepte zu vermeiden sowie das Interview neutral zu moderieren. Das Interview dient ausschließlich der Erläuterung 


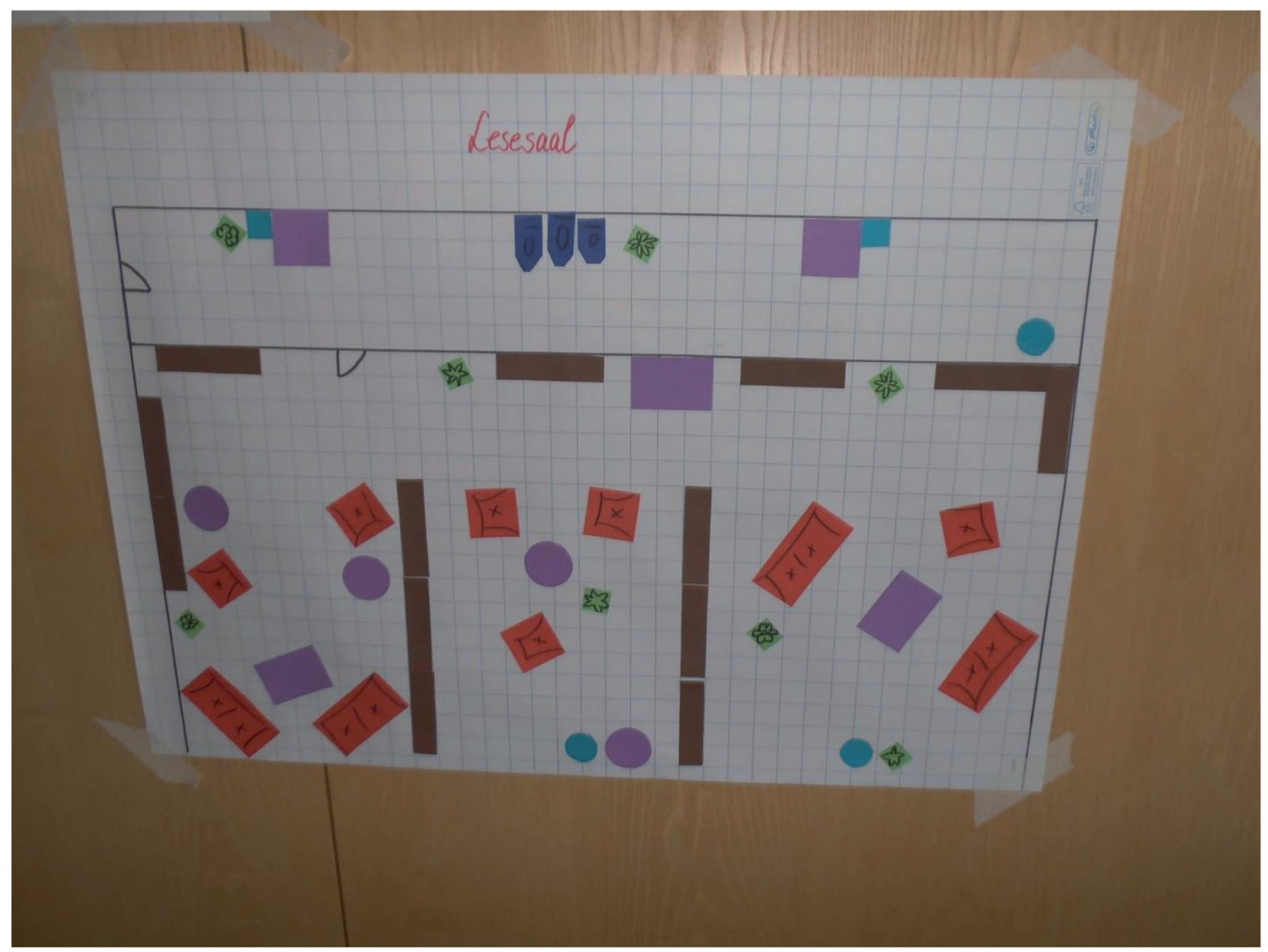

Abb. 6: Schnappschuss aus einem Design-Workshops der UB Rostock: Zu sehen ist der „ideale Bibliotheksarbeitsplatz“

der Skizzen durch deren Designer. Darin integriert werden können auch bibliotheksseitige Nachfragen $\mathrm{zu}$ für diese Bibliothek wichtigen Aspekten wie $\mathrm{Zu}$ schnitt von Gruppenarbeitszonen, Lage zu Peripherieräumen (WC, Drucker/Scanner, Garderobe, Servicetheken).

- Dokumentation und Auswertung: Die Workshopergebnisse werden aus Raumskizzen, die anschließend abfotografiert werden, sowie aus deren interviewbasierten verschriftlichten Erläuterungen bestehen. Die UB Rostock hat ihre Workshopergebnisse mit einer Tonspur versehen auf Youtube veröffentlicht. ${ }^{3}$ Sollten die Interviews aufgezeichnet werden, z.B. um sie später transkribieren zu können, ist das Einverständnis der Bibliotheksnutzer dafür nötig. Möglichst zeitnah dazu sollte eine bibliotheksseitige Auswertungsrunde fol-

3 https://www.youtube.com/watch?v=QHDf5j07L60. gen, in der Kollegen aus verschiedenen Bereichen (!) diese Skizzen leitfragenorientiert auswerten.

\subsubsection{Vorteile}

Da als Ergebnis Raumskizzen entstehen, sind DesignWorkshops besonders attraktiv für die Suche nach (nutzerorientierten) Raumgestaltungsmöglichkeiten: Anstelle allein verbalisiert vorliegender Lernraumnutzerbedürfnisse, liefern Nutzer hier von ihnen in Raumskizzen übersetzte Lernraumbedürfnisse, die zudem komplexe Nutzungsvorgänge abbilden können (z. B. abgestimmte Verkehrswege, Kombination von Einzel- mit Gruppenarbeitsphasen, Raumaufteilungswünsche). Auch der spielerisch-fiktionale Charme eröffnet einen potenziell weiten und ergiebigen Ideenspielraum für Anregungen für eine nutzerorientierte Ausgestaltung von Lernräumen. Und schließlich können Design-Workshops implizit als besonderes Nutzerkom- 
munikationsformat dienen, um mit Nutzern allgemein ins Gespräch zu kommen.

\subsubsection{Risiken}

Eine Gefahr besteht darin, Design-Workshops methodisch $\mathrm{zu}$ überfordern: Mit ihnen ist es weder sinnvoll noch praktikabel, repräsentative Ergebnisse anzustreben. Dagegen sind die Workshopergebnisse ideal für Gestaltungsanregungen und erste Schlüsse auf aktuelle Lernraumbedürfnisse. Ein eher abstraktes Risiko für den erfolgreichen Einsatz dieser Methode ist die bibliotheksseitige Überinterpretation dieser visualisierten Raumbedürfnisse bzw. Raumskizzen, z.B. kann eine darin eingezeichnete Minibar für eine Minibar stehen, aber auch als Sinnbild für eine (gewünschte) Erlaubnis der Getränkemitnahme an den Arbeitsplatz oder für ein Verwohnlichungswunsch des Arbeitsplatzes. Das im Design-Workshop eingebaute Teilnehmerinterview soll den Deutungsspielraum reduzieren.

\subsubsection{Praxistipps}

- Workshopleitung: Um Brücken zu schlagen, können sich Peers besser eignen als Bibliotheksmitarbeiter, z. B. (Projekt-)Studierende als Workshopleiter bei studentischen Bibliotheksnutzern.

- Gruppen- oder Einzelworkshops: Die Designworkshops können auch aus nur einem oder zwei Teilnehmern bestehen. Das erweist sich zudem vorteilhaft, wenn Interviews aufgezeichnet und transkribiert werden sollen (Zuordnung von Stimmen zu Aussagen).

- Lernraumanregungen: Hilfreich kann werden, einen Teil der Workshopteilnehmer zuvor beliebige andere Lernräume wertfrei zu vermitteln a) als Anregung für die bevorstehende Aufgabe und b) um deren Wissen, wie Lernräume in Bibliotheken gestaltet werden können, zu erweitern.

- Grundriss: Bewährt hat sich, den Workshopteilnehmern die Wahl zu lassen: entweder einen - realen oder fiktiven - Grundriss vorzugeben oder ihnen zu überlassen, einen Flächengrundriss zu entwerfen.

\subsection{Möbelcasting}

\subsubsection{Zweck}

Büro- und vor allem Lernräume in Hochschulbibliotheken erfüllen ihre Funktion zu einem großen Teil über ihr Mobiliar. Bevor Mobiliar für Nutzerarbeitsplätze beschafft wird, sollte ein Möbelcasting vorangehen: $\mathrm{Zu}$ beschaffendes Mobiliar wird von denen, die es benutzen sollen, testweise und über einen angemessenen Zeitraum genutzt und evaluiert, und das möglichst dort, wo es künftig genutzt werden soll. Evaluiert wird, inwieweit das Mobiliar den Zwecken genügt, zu denen es beschafft werden soll. Dazu können z.B. Gruppenarbeitsmöbel, akustisch wirksame Trennwände, Wasserspender oder Präsentationsmobiliar gehören.

\subsubsection{Methodischer Kerngedanke}

Aus der Perspektive der Nutzerorientierung bzw. des Partizipativen Gestaltens ist es eine Selbstverständlichkeit, Mobiliarbeschaffungen von denen mitentscheiden $\mathrm{zu}$ lassen, die es später nutzen werden. Dabei werden - analog zum Casting - mehrere Produkte, die ein und dieselbe Funktion übernehmen sollen (z. B. lärmreduzierende Gruppenarbeitsplatzeinheiten), getestet und evaluiert.

\subsubsection{Vorgehensweise}

Nachdem definiert wurde, wie hoch der Beschaffungsetat dafür sein kann und für welche konkrete (Lernraum-)Aufgabe Mobiliar beschafft werden soll, z. B. flexibles Gruppenarbeiten in lärmberuhigter Zone, wird bibliotheksseitig ein Katalog von ersten Kriterien erarbeitet, die ein potenziell geeignetes Mobiliar an einem bestimmten Standort erfüllen muss, z. B. formale Kriterien wie Brandschutz-, Akustikklassen, Scheuertouren (Scheuerbeständigkeit), Kosten-, Lieferzeitrahmen. Daneben müssen inhaltliche Kriterien wie runde Tischfläche, Rollbarkeit, Kombinierbarkeit $\mathrm{zu}$ verschiedenen Tischformen, kontrastarme Oberflächenfarbe fixiert werden. Diese Kriterien basieren idealerweise auf ersten eigenen oder allgemeinen Nutzerforschungen. Anhand dessen beginnt eine erste Vorauswahl von für die je eigene Bibliothek infrage kommenden Möbellösungen zusammen mit fraglichen Herstellern bzw. Zwischenhändlern, mit denen schließlich der Startzeitpunkt und die Testdauer vereinbart werden. Nicht alle Hersteller bzw. Zwischenhändler wollen oder können Möbeltests ermöglichen. 


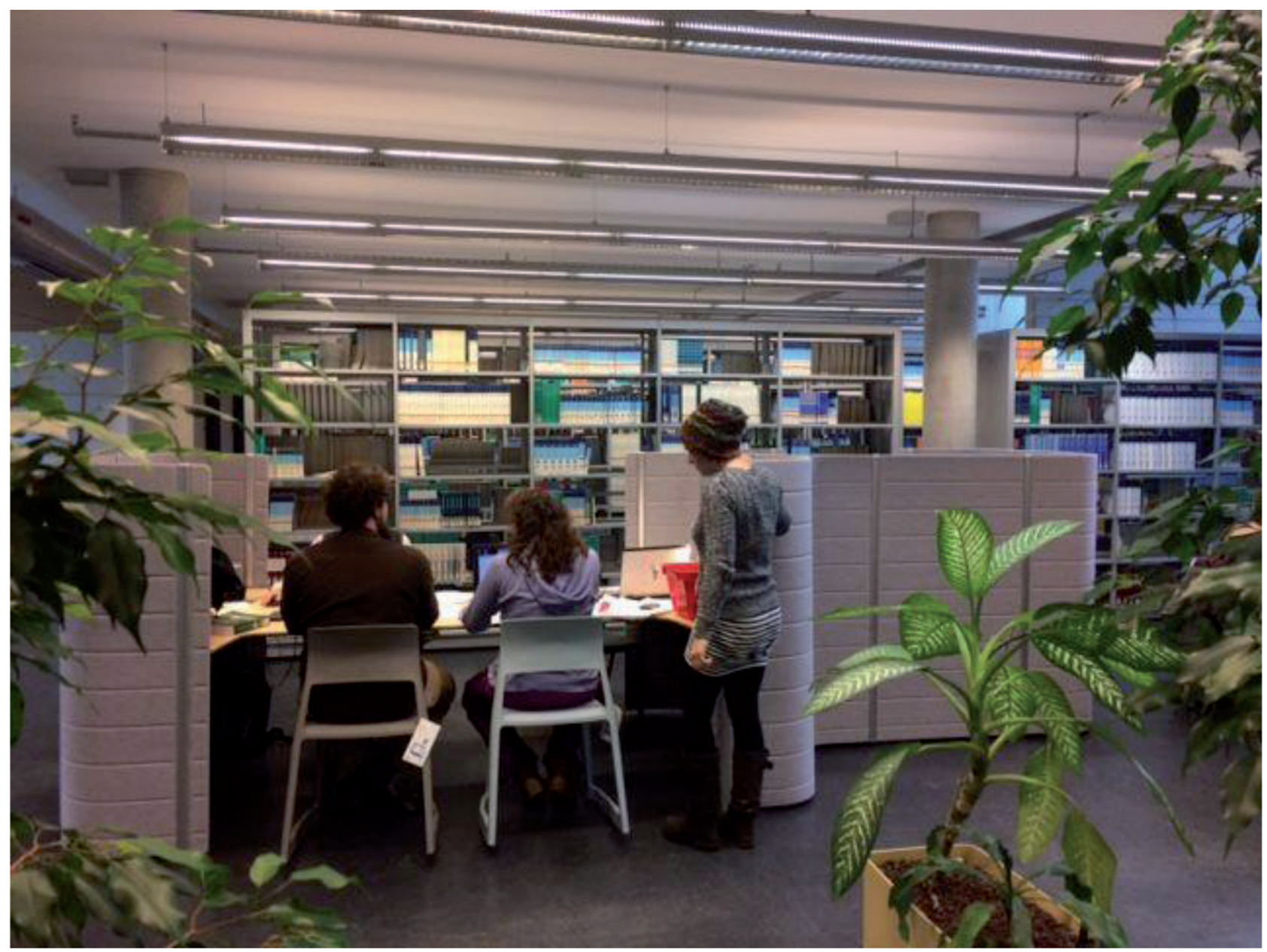

Abb. 7: Studierende an der UB Rostock testen Gruppenarbeitsmobiliar der Fa. Vitra (Workbay)

Die Öffentlichkeitsarbeit für ein Möbelcasting-Projekt hat vor allem die Funktion, die Zielgruppe zu einer regen Testnutzung und Feedback zu ermuntern sowie - um Missverständnisse zu vermeiden - die Zielstellung dieses Projekts zu erläutern.

Parallel dazu wird ein niedrigschwelliges Evaluationsinstrument konzipiert, z. B. ein einfacher Papierfragebogen mit ein bis zwei Zufriedenheitsfragen und einem großen offenen Kommentarfeld. Letzteres hat sich an der UB Rostock als sehr ergiebig erwiesen: Es enthielt detaillierte Ergebnisse, wann was aus Sicht von Studierenden geeignet ist.

\subsubsection{Vorteile}

Möbelcastings haben auch den Vorteil, dass sowohl das bibliotheksseitige aktive Bemühen um die Lernraumgestaltung als auch die Nutzerpartizipation räumlich sichtbar werden. Zudem haben sie eine hohe Akzeptanz unter studentischen Bibliotheksnutzern, so dass eine Ergebnisgarantie ebenso wie eine hohe Mitwirkungsbereitschaft erwartet werden darf. Und im Idealfall liefern Möbelcastings auch einen Anforderungskatalog für Lernraummobiliar: Die wahrscheinlich vielen verbalen Rückmeldungen können wertvolle detaillierte Hinweise enthalten, wann was z. B. ein praktikabler Stuhl, Sichtschutz oder Gruppenarbeitstisch ist.

\subsubsection{Risiken}

Dieses Verfahren scheint weitgehend risikolos. Als etwas hinderlich kann sich erweisen, dass positiv evaluiertes Mobiliar unerwartet nicht beschafft werden kann. Bibliotheksnutzer können sich rasch an Mobiliar gewöhnen. 


\subsubsection{Praxistipps}

- Beschaffung: Zum einen, um eine breite Lieferantenauswahl zu erreichen, zum anderen, um evtl. bestehende vergaberechtliche Hürden $\mathrm{zu}$ räumen (z.B. kann Bemustern zuvor eine Ausschreibung erfordern), empfiehlt sich, dieses Projekt zuvor mit der Beschaffungsstelle o. ä. abzustimmen.

- Vorbeugender Brandschutz, Reinigung: Abhängig vom künftigen Standort muss das Mobiliar bestimmte Sicherheitsbestimmungen erfüllen, die sich auch auf den Beschaffungspreis auswirken. Eine vorangehende Abstimmung mit dem Sicherheitsbeauftragen o.ä. und dem Gebäudemanagement ist unumgänglich, nicht zuletzt, um ggf. die vertraglichen Routinereinigungen von Mobiliar zu erweitern.

- Hersteller, Lieferanten: Nicht alle Hersteller oder Lieferanten sind bereit, ihr Mobiliar zu längeren Tests zur Verfügung zu stellen. Ein Anreiz für sie könnten die erwarteten Evaluationsergebnisse sein, die die Bibliothek nach dem Möbelcasting ihnen in Aussicht stellen kann.

\section{Fazit}

Partizipative Gestaltung bzw. Nutzerforschung ist aufwändig, ja. Aber sie lohnt sich: Zum einen fühlen sich die Bibliotheksnutzer glaubhaft ernstgenommen, zum anderen kann der Ergebnisertrag sehr hoch sein, d.h., die Bibliothek kann ihre Nutzer verstehen lernen und von den empirischen und kreativen Ergebnissen sowohl kurz- als auch mittelfristig zehren. Und nicht zuletzt nimmt die bibliothekseigene Lösungsexpertise deutlich zu, das Spektrum möglicher Lösungsansätze wird erweitert. Im o.g. Projekt waren die Lösungsideen der Studierenden mal überraschend, mal klassisch, mal unorthodox - aber überwiegend hilfreich und realisierbar. Ähnliche Erfahrungen haben inzwischen auch andere Hochschulbibliotheken gemacht. Um dieses Potenzial nachhaltig abzusichern, arbeitet die UB Rostock nun an einer Verstetigung in Form eines zeitgemäßen Konzepts für einen studentischen Nutzerbeirat - für mehr und größere Gestaltungsspielräume!

\section{Literaturverzeichnis}

Ilg, Jens; Zepf, Robert (2016): Partizipatives Gestalten. In: Praxishandbuch Bibliotheksbau: Planung - Gestaltung - Betrieb, hg.v. Petra Hauke und Klaus Ulrich Werner, 295-307. Berlin, Boston: De Gruyter Saur (De Gruyter Reference).

Vogel, Bernd; Woisch, Andreas (2013): Orte des Selbststudiums: Eine empirische Studie zur zeitlichen und räumlichen Organisation des Lernens von Studierenden. Hannover: HIS (Forum Hochschule: 2013,7$)$.

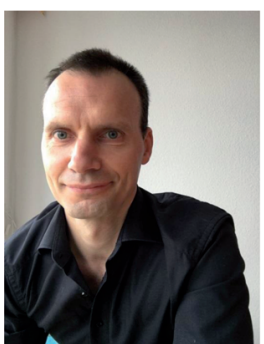

Jens IIg

Leiter Bereichsbibliothek Innenstadt Fachreferent Bildungswissenschaft, Musikwissenschaft, Philosophie, Psychologie Universitätsbibliothek Rostock

August-Bebel-Str. 28

D-18051 Rostock jens.ilg@uni-rostock.de 